\title{
Sphere Drag in Flows of Almost-Free Molecules
}

\author{
Vi-Cheng Liu, Sing-Chin Pang, and Howard Jew \\ Department of Aeronautical and Astronautical Engineering, \\ The University of Michigan, Ann Arbor, Michigan
}

(Received 5 June, 1964; final manuscript received 5 February 1965)

\begin{abstract}
A kinetic theory of sphere drag in the transition flows, based on the Boltzmann equation for the Maxwellian molecules, is presented. The exact binary collision integral, in the first order Knudsen iteration, is expanded as a function of Hermite polynomials in molecular velocity. The drag of a sphere at free stream temperature with molecular diffuse reflection is calculated. The results agree well with Millikan's measured values over a wide range of Knudsen number $(0.5<\lambda / d<10)$. It is found that the sphere drag of the almost-free molecular flows normalized by the corresponding collisionless drag is essentially independent of the speed ratio for the present range of calculations.
\end{abstract}

\section{INTRODUCTION}

$\mathrm{T}$ HE recent interest on sphere drag in a rarefied gas has been stimulated, partly at least, by the advent of the earth satellite experiments and the upper atmosphere measurements. The theory of sphere drag in the extremely rarefied medium such that the state of free molecules ${ }^{1}$ exists, is well known provided the reflection mechanisms of the incident molecules on the solid surface are adequately represented by accommodation coefficients of Maxwell and Knudsen.

Numerous attempts have been made recently in the determination of sphere drag for the state slightly less rarefield than free molecules. This has been called the near-free molecular flows (or almost-free molecular flows). Many of these attempts are limited either to the flows of extremely high speeds $^{2,3}$ or to the extremely low speeds. ${ }^{4}$ These restrictions have been introduced to make the analyses tractable. In the present study, an effort is made to remove the limitations imposed in previous analyses on the speed of the sphere. The purpose of the present work is twofold: (1) to obtain the sphere drag in a rarefied atmosphere which is of utmost interest in upper atmosphere measurements, (2) to understand the fundamental nature of the transition flows with a model which is realistic enough such that accurate drag measurements, either free flight or laboratory, can be made in the near future in order to make meaningful comparisons.

It is a matter of simple dimensional analysis to show that the flows around geometrically similar

\footnotetext{
${ }^{1}$ G. N. Patterson, Molecular Flow of Gases (John Wiley \& Sons, Inc., New York, 1956)

${ }^{2}$ D. R. Willis, Rand Report R-339 (1959).

${ }^{3}$ R. M. L. Baker and A. F. Charwat, Phys. Fluids 1, 73 (1958).

${ }^{4}$ Z. Szymanski, Arch. Mech. Stos (Warsaw) 8, 449 (1956); 9, $35(1957)$.
}

bodies without the influence of external force are dynamically similar, provided the flows have equal speed ratio $s$ and Kundsen number $\mathrm{Kn}$, respectively.

Experimental results of sphere drags corresponding to the Knudsen numbers of the near-free molecular flows rarely exist except for the excellent measurements by Millikan ${ }^{5}$ which covers a wide range of Knudsen number $(0.01<\mathrm{Kn}<10)$ at however extremely low speed ratios $\left(s<10^{-5}\right)$. Millikan's data for which the experimental conditions were clearly defined may serve as important check points for theory of sphere drag at asymptotically low speed ratios.

The elementary kinetic theory of the transition flows based on collision statistics ${ }^{3,6}$ can only give a gross quantitative answer to the problem of interest as was painstakingly cautioned. ${ }^{6} \mathrm{~A}$ rigorous theory must start with the Boltzmann equation. The present study on sphere drag gives the first-order $\left(\mathrm{Kn}^{-1}\right)$ iteration of the Boltzmann equation for the Maxwellian molecules. ${ }^{7}$ The basic approach is similar to that of Szymanski except that we preserve the nonlinear terms of speed ratio in the Boltzmann collision integral which is essential in the treatment of high speed flows. The inclusion of these nonlinear terms considerably complicates the collision integral analysis.

\section{FORMAL ITERATION OF THE BOLTZMANN EQUATION}

Consider a gas with Maxwellian molecules ${ }^{7}$ of mass $m$, number density $n_{\infty}$, and with the most probable velocity $w_{\infty}$. To describe the molecular distribu-

${ }_{5}^{5}$ R. A. Millikan, Phys. Rev. 22, 1 (1923).

6 V. C. Liu, J. Aeron. Sci. 25, 779 (1958); also J. Fluid Mech. 5, 481 (1959).

7 S. Chapman and T. G. Cowling, Mathematical Theory of Non-Uniform Gases (Cambridge University Press, New York, 1951). 
tion surrounding a spherical body of diameter $d$ placed in a free stream of velocity $\mathbf{u}_{\infty}$, we introduce a molecular distribution function $F(\mathbf{c}, \mathrm{r})$ for the molecules at point $\mathbf{r}$ with velocity $\mathbf{c}$ in dimensionless form with displacement and velocity expressed in units of $\frac{1}{2} d$ and $w_{\infty}$, respectively. If the differential collision cross section for the molecular collisions can be expressed in units of the momentum cross section $B_{1}$ for Maxwellian molecules, ${ }^{8}$ we can write the steady state Boltzmann equation for Maxwellian molecules in dimensionless form as follows:

$$
\begin{aligned}
\mathrm{c} \cdot \frac{\partial F}{\partial \mathrm{r}}=\frac{d n_{\infty} B_{1}}{2 w_{\infty}} \int d \mathrm{c}_{1} \int_{0}^{2 \pi} d \epsilon \int_{0}^{\pi / 2} d \theta \\
\cdot B(\theta)\left(F^{\prime} F_{1}^{\prime}-F F_{1}\right),
\end{aligned}
$$

where

$B_{1}=0.343(2 K / m)^{\frac{1}{2}}$ (intermolecular force $\left.=K / \eta^{5}\right)$ $B(\theta) d \theta d \epsilon=b d b d \epsilon / 0.343\left(b=\right.$ impact parameter $\left.{ }^{7}\right)$.

A physical interpretation can be given to the dimensionless constant in front of the integrals in Eq. (2.1). If we define a mean free path $\lambda$ based on the momentum cross section $B_{1}$ and use the definition of viscosity $\mu$ from transport theory for Maxwellian molecules, namely ${ }^{9}$

$$
\mu=k T / 6 B_{1}=0.491(4 / \pi)^{\frac{1}{2}} m n_{\infty} w_{\infty} \lambda
$$

we can rewrite (2.1) as

$$
\begin{aligned}
\mathrm{c} \cdot \frac{\partial F}{\partial \mathrm{r}}=0.075 \frac{d}{\lambda} \int d \mathrm{c}_{1} \int_{0}^{2 \pi} d \epsilon \int_{0}^{\pi / 2} d \theta \\
\cdot B(\theta)\left(F^{\prime} F_{1}^{\prime}-F F_{1}\right),
\end{aligned}
$$

in other words, this dimensionless constant of interest is inversely proportional to the Knudsen number $(\lambda / d)$.

For the studies of momentum transfer in flows at high Knudsen number, it is permissible to expand the distribution function $F$ formally into a power series in $d / \lambda$,

$$
F=F^{(0)}+(d / \lambda) F^{(1)}+\cdots
$$

A word of caution should be noted concerning the appropriateness of the asymptotic expansion in Knudsen number as shown in Eq. (2.4). It has been found, ${ }^{10}$ e.g., that with such expansion in the study

${ }^{8}$ H. Grad, Commun. Pure Appl. Math. 2, 325 (1949). [Note: the definition of $B(\theta)$ is different from Grad's by a factor of $B_{1}$ ].

${ }_{9}$ For the Maxwellian molecules, $B_{1}=B_{1}^{(n)}$ following the notations used in Ref. 8.

${ }_{10} \mathrm{C}$. S. Wang Chang and G. E. Uhlenbeck, University of Michigan ERI Report M999 (1953); D. R. Willis, thesis, Princeton University (1959). of the shear flow between parallel plates, a singularity appears in the expression for flow velocity. This does not, however, suggest that similar difficulty will necessarily appear in every flow study with the method in question. The following sequence of equations follows, after the substitution of Eq. (2.4) into Eq. (2.1) and a rearrangement of terms in powers of $d / \lambda$.

$$
\begin{gathered}
\mathrm{c} \cdot \partial F^{(0)} / \partial \mathrm{r}=0 \\
\mathbf{c} \cdot \frac{\partial F^{(1)}}{\partial \mathrm{r}}=0.075 \frac{d}{\lambda} \int d \mathbf{c}_{1} \int_{0}^{2 \pi} d \epsilon \int_{0}^{\pi / 2} d \theta \\
\cdot B(\theta)\left(F^{(0)} F_{1}^{(0) \prime}-F^{(0)} F_{1}^{(0)}\right) \cdots .
\end{gathered}
$$

It is observed that the left-hand side of Eq. (2.5) is the derivative of $F^{(0)}$ in the direction of the vector $c$ in the molecular phase space. At each point in this space $(c, r)$, this vector points in the direction of the molecular trajectory through that point, which is also the characteristic curve of the equation. Hence if $q$ denotes arc length along a trajectory, Eqs. (2.5) and (2.6) become respectively

$$
\begin{aligned}
& c d F^{(0)} / d q=0, \\
& c d F^{(1)} / d q=0.075(d / \lambda) E(\mathbf{r}, \mathbf{c}),
\end{aligned}
$$

where $E(\mathbf{r}, \mathbf{c})$ denotes the multiple integral in Eq. (2.6), the physical significance of which is discussed later.

Since Eq. (2.7) states that the function $F^{(0)}$ is constant along a trajectory, it is the distribution function for the free molecular, or collisionless, flows when appropriate boundary conditions of interest have been satisfied. Equation (2.8), which contains a collision term expressed in terms of the free molecular distribution $F^{(0)}$, may be considered as the kinetic equation of flow of the almost free molecules. ${ }^{11}$ It may be noted that the previous analyses made on the flows of the almost-free molecules ${ }^{3,6}$ are, in essence, some macroscopic moments of Eq. (2.8) for mass and momentum fluxes, etc., after drastic simplifications have been made to the collision integral $E$.

It is further noted that in view of the mathematical structure of Eqs. (2.7) and (2.8), the analysis of the rarefied gas invariably involves integrations along the characteristic curves when the flow fields need to be mapped. In the case of the free molecular flows, this is quite similar to the problem of geometrical optics. In fact, the line-of-sight principle will be adopted in mapping the distribution $F^{0}(\mathbf{r}, \mathbf{c})$.

${ }^{11}$ V. C. Liu, University of Michigan ORA Report 0288511-F (1962). 


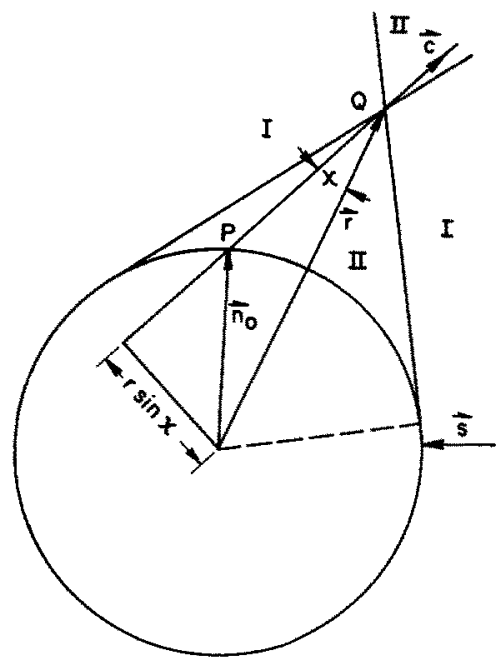

Fig. 1. Sphere-cone geometry.

\section{ZEROTH-ORDER APPROXIMATION TO THE MOLECULAR DISTRIBUTION}

As a prelude to the analysis of flows of the almost free molecules, we must first obtain the solution $F^{(0)}$ to the collisionless Eq. (2.7) with boundary conditions prescribed on the sphere and upstream. This constitutes our zeroth-order approximation to the problem.

It is noted that the aggregation of molecules at any point ( $r$ ) in a free molecular flow field must come from either of two sources: the free stream and the reflected stream from the solid surface. Thus we simply express $F^{(0)}$ in terms of functions $f(\mathbf{r}, \mathbf{c})$ and $g(\mathbf{r}, \mathbf{c})$ as follows:

$$
F^{0}=f+g
$$

where

$$
\begin{gathered}
f=\pi^{-\frac{3}{3}} \exp \left[-(\mathrm{c}-\mathbf{s})^{2}\right] \text { for all regions, } \\
g=0 \text { for region I (see Fig. 1), } \\
=\pi^{-\frac{3}{3}} n(P) \exp \left(-c^{2}\right)-\pi^{-\frac{1}{2}} \exp \left[-(\mathbf{c}-\mathbf{s})^{2}\right] \\
\text { for region II, }
\end{gathered}
$$

where $\mathbf{s}$ denotes the free stream velocity in units of $w_{\infty}$, i.e., $s=u_{\infty} / w_{\infty}$ and the equivalent number density $n(P)$ of reflected molecules at point $P$ on the sphere (see Fig. 1) is given by ${ }^{1}$

$$
\begin{aligned}
n(P)=\exp \left[-\left(\mathbf{s} \cdot \mathbf{n}_{0}\right)^{2}\right] & -\pi^{\frac{1}{2}} \mathbf{s} \cdot \mathbf{n}_{0} \\
& +\pi^{\frac{t}{s}} \mathbf{s} \cdot \mathbf{n}_{0} \text { erf }\left(\mathbf{s} \cdot \mathbf{n}_{0}\right) .
\end{aligned}
$$

In (3.5) $\mathrm{n}_{0}$ denotes a unit vector as shown in Fig. 1 . The subscript 0 always designates a unit vector in the direction of the vector it subscripts, e.g., $\mathbf{s}_{0}=$ $\mathbf{s} /|\mathbf{s}|$, etc.
It can be shown, by referring to Fig. 2 that

$$
\begin{aligned}
\mathbf{n}_{0} \cdot \mathbf{s}_{0} & =\mathbf{s}_{0} \cdot \mathbf{r} \\
& -\left(\mathbf{s}_{0} \cdot \mathbf{c}_{0}\right)\left[\left(\mathbf{r} \cdot \mathbf{c}_{0}\right)-\left(1-r^{2} \sin ^{2} \chi\right)^{\frac{1}{2}}\right] .
\end{aligned}
$$

In the present study the function $n(P)$ is approximated by a linear function with $s$-dependent coefficients $\alpha(s)$ and $\beta(s)$ as follows:

$$
n(P)=\alpha s x+\beta,
$$

where $x=\mathbf{s}_{0} \cdot \mathbf{n}_{0}$ and the parameters $\alpha, \beta$ are to be determined by considering the conservation of particles for the upstream and downstream semispherical surfaces respectively (see Appendix I).

\section{MATHEMATICAL REPRESENTATION OF THE FIRST-ORDER COLLISION EFFECT}

In the interest of aerodynamic drag, we are concerned only with a few lower moments of the distribution function $F^{(1)}$ rather than the function itself. Accordingly, the present approach dwell primarily with the determination of moments for $F^{(1)}$ from the use of Eq. (2.8). The fact, however, that the distribution $F^{(0)}$ appearing in the integrand of the collision integral $E$ is a discontinuous functionan inherent feature of the free molecular distribution-makes it unfruitful to use the moment-generation technique of $\mathrm{Grad}^{8}$ which appears effective for the near-continuum flows only. ${ }^{12}$

It is observed that the right-hand side of Eq. (2.8) can be interpreted as the distribution function of molecules having collisions in the neighborhood of the point (r). In more precise statement, it can be said that $0.075(d / \lambda) E(\mathbf{r}, \mathbf{c}) d \mathbf{r} d \mathbf{c}$ represents the net rate of change of the number of molecules situated in the region $\mathbf{r}, \mathbf{r}+d \mathbf{r}$ and having velocities between c and $\mathbf{c}+d \mathbf{c}$. Note that collisions have the tendency

Fra. 2. Field coordinates.

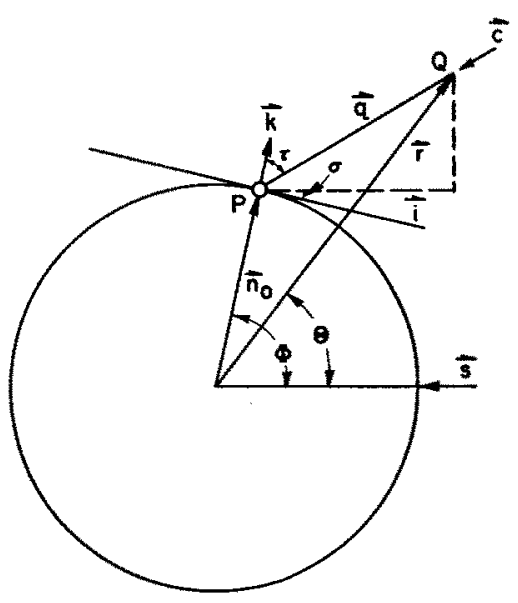

${ }^{12}$ R. Goldberg, thesis, New York University (1954). 
to randomize the molecular arrangement hence to smooth out the discontinuity in molecular arrangement in the molecular phase space. It is therefore postulated that for the almost-free molecules the collisional distribution $E(\mathbf{r}, \mathbf{c})$ should be better suited for representation, compared to $F$ for instance, by a series of three dimensional Hermite polynomials $H_{i j k}^{(n)} \ldots$ (c) which has been used by $\mathrm{Grad}^{8}$ for representing the distribution $F(\mathbf{r}, \mathbf{c})$ in the near-continuum flows. This must be considered as the fundamental hypothesis of the present approach.

\section{A. Series Expansion for Distribution $E(\mathbf{r}, \mathbf{c})$}

It is assumed that

$$
E(\mathbf{r}, \mathbf{c})=(2 \pi)^{-\frac{3}{2}} \exp \left[-\left(\frac{1}{2} c^{2}\right)\right] \sum_{n=0}^{\infty} a_{i j k}^{(n)} \ldots H_{i j k}^{(n)} \ldots
$$

$$
(i, j, k \text { ranges from } 1 \text { to } 3),
$$

where the coefficients $a_{i j k}^{(n)} \ldots$, symmetrical tensors of the $n$th order, are functions of $\mathrm{r}$, and $H_{i j k}^{(n)} \ldots$, the three-dimensional Hermite polynomials of the $n$th order, are functions of c. The coefficients are, in turn, expressible as

$$
a_{i j k}^{(n)} \ldots=\frac{1}{n !} \int d \mathbf{c} H_{i j k}^{(n)} \ldots E(\mathbf{r}, \mathbf{c}) .
$$

Equation (4.2), after the substitution of $F^{(0)}=f+g$ from (3.1) and $E(\mathbf{r}, \mathrm{c})$ from (2.6) and (2.8), also the use of symmetry relations for the binary collision integral, ${ }^{7}$ becomes

$$
\begin{aligned}
a^{(n)}=\frac{1}{n !} \iint d \mathbf{c} d \mathbf{c}_{1} & \int_{0}^{2 \pi} d \epsilon \int_{0}^{\pi / 2} d \theta \\
& \cdot B(\theta)\left[H^{(n)}\right]\left(g_{1} f+\frac{1}{2} g_{1} g\right),
\end{aligned}
$$

where

$$
\left[H^{(n)}\right]=H^{(n)}\left(\mathbf{c}^{\prime}\right)+H^{(n)}\left(\mathbf{c}_{1}^{\prime}\right)-H^{(n)}(\mathbf{c})-H^{(n)}\left(\mathbf{c}_{1}\right) .
$$

Subscripts $i j k \cdots$ have been dropped since the order of tensor is designated by superscript $(n)$.

From conservations of momentum and energy in elastic collisions of molecules, we obtain the velocities after collision (see Fig. 1)

$\mathbf{c}_{1}^{\prime}=\mathbf{c}_{1}+h \cos \theta \mathbf{k}_{0}, \quad \mathbf{c}^{\prime}=\mathbf{c}-h \cos \theta \mathbf{k}_{0}$,

where $h$ is the velocity of approach in the center of mass system and $\mathbf{k}_{\mathbf{0}}$ is the unit vector pointing to the direction of interaction force that changes the vector $\mathbf{h}$ to $\mathbf{h}^{\prime}$. The substitution of (4.5) in (4.4) and the use of the identity:

$$
H^{(n)}(\mathbf{a}+\mathbf{b})=\sum_{j=0}^{n} H^{(n-i)}(\mathbf{a}) \mathbf{b}^{i}
$$

leads to

$$
\begin{array}{r}
{\left[H^{(n)}\right]=\sum_{i=1}^{n}\left\{H^{(n-i)}\left(\mathbf{c}_{1}\right)+(-1)^{i} H^{(n-i)}(\mathbf{c})\right\}} \\
\cdot \cos ^{i} \theta h^{j} \mathbf{k}_{0}^{j} .
\end{array}
$$

Further development of (4.3) involves integrals of the following type:

$$
\begin{gathered}
\int_{0}^{2 \pi} d \epsilon \mathbf{k}_{0}^{j}=\sum_{m=0}^{N(j / 2)} \mathbf{h}_{0}^{(j-2 m)}\left(\boldsymbol{\delta}-\mathbf{h}_{0}^{2}\right)^{m} 2^{1-m} \pi(-1)^{i} \\
\cdot \cos ^{j-2 m} \theta \sin ^{2 m} \theta / m !
\end{gathered}
$$

where $N\left(\frac{1}{2} j\right)$ denotes the highest integer not greater than $\frac{1}{2} j ; \mathbf{\delta}$, the unit tensor or Kronecker delta $\delta_{i j}$ with $i, j$ omitted. ${ }^{8}$

Define

$$
B_{i m}=\pi \int_{0}^{\pi / 2} \sin ^{2 m} \theta \cos ^{2 i-2 m} \theta B(\theta) d \theta .
$$

The expression (4.3), after the use of (4.6), (4.7), and (4.8), becomes

$$
\begin{aligned}
& a^{(n)}=\frac{1}{n !} \sum_{j=1}^{n} \sum_{m=0}^{N(j / 2)} \iint d \mathbf{c} d \mathbf{c}_{1} \\
& \cdot\left\{H^{(n-j)}(\mathbf{c})+(-1)^{i} H^{(n-j)}\left(\mathbf{c}_{1}\right)\right\} \mathbf{h}^{j-2 m}\left(h^{2} \mathbf{\delta}-\mathbf{h}^{2}\right)^{m} \\
& \cdot B_{j m}\left(2^{1-m} g_{1} f+2^{-m} g_{1} g\right) / m !
\end{aligned}
$$

So far, the expansion has been given to the $n$th order. In the evaluation for collision effects, we shall truncate the series beyond $n=2$; the mathematical effort of higher-order terms becomes prohibitively heavy and is probably not rewarding because, as it will be shown, the present approximation appears satisfactory.

\section{B. Evaluation of the Coefficient $a^{(2)}$}

A careful study of (4.8) will show that the final evaluation of the coefficient $a^{(2)}$ amounts to the calculations of the following types of moment integrals:

$$
\text { (1) }{ }^{l} f^{n}=\int \mathbf{c}^{n} c^{l} f d \mathbf{c}
$$

over the whole velocity space, which can be integrated without much trouble. The results of the first few moment integrals $(n \leq 2)$ are as follows:

$$
\begin{aligned}
& { }^{0} f^{0}=1 ;{ }^{0} f^{1}=\mathbf{s} ;{ }^{0} f^{2}=\mathbf{s}^{2}+\frac{1}{2} \delta ;{ }^{2} f^{0}=s^{2}+\frac{3}{2} \\
& \text { (2) }{ }^{l} g^{n}=\int \mathbf{c}^{n} c^{l} g d \mathbf{c}
\end{aligned}
$$

over a conical domain subtended at the point $\mathbf{r}$ by the sphere of diameter $d$. (see Fig. 1) To accomplish 
the integration we divide the $g$-function into three parts

$$
g=g_{1}+g_{2}-g_{3}
$$

where

$g_{1}=\pi^{-\frac{3}{2}}\left[\alpha s\left(\mathbf{s}_{0} \cdot \mathbf{r}\right)+\beta\right] \exp \left(-c^{2}\right)$

$g_{2}=-\pi^{-\frac{3}{2}} \alpha s\left(\mathbf{s}_{0} \cdot \mathbf{c}_{0}\right)\left\{\mathbf{r} \cdot \mathbf{c}_{0}-\left(1-r^{2} \sin ^{2} \chi\right)^{\frac{1}{2}}\right\} \exp \left(-c^{2}\right)$

$g_{3}=\pi^{-\frac{3}{2}} \exp \left[-(\mathrm{c}-\mathrm{s})^{2}\right]$.

These moment integrals have been evaluated and the results are as follows:

$$
\begin{aligned}
{ }^{i} g_{1}^{n}= & (\alpha \mathbf{S} \cdot \mathbf{r}+\beta) \pi^{-\frac{3}{2}} \\
& \cdot \Gamma\left(\frac{n+l+3}{2}\right) \sum_{m=0}^{N(j / 2)} \mathbf{r}_{0}^{n-2 m}\left(\boldsymbol{\delta}-\mathbf{r}_{0}^{2}\right)^{m} \alpha_{n m},
\end{aligned}
$$

where

$\alpha_{n m}=\pi \int_{0}^{\sin { }^{-1} 1 / r} 2^{-m} \cos ^{n-2 m} \chi \sin ^{2 m+1} \chi d \chi / m !$

and

$$
\begin{gathered}
{ }^{l} g_{2}^{n}=-\pi^{-\frac{y}{2}} \mathbf{\Gamma}\left(\frac{n+l+3}{2}\right) \\
\cdot \alpha \mathbf{s} \cdot \sum_{m=0}^{N\left(\frac{1}{2}(n+1)\right)} \mathbf{r}_{0}^{n+1-2 m}\left(\boldsymbol{\delta}-\mathbf{r}_{0}^{2}\right)^{m} \beta_{n m}, \\
\beta_{n m}=\pi \int_{0}^{\sin { }^{-1}} 2^{-m}\left[r \cos \chi-\left(1-r^{2} \sin ^{2} \chi\right)^{\frac{1}{2}}\right. \\
\left.\cdot \sin ^{2 m+1} \chi \cos ^{n+1-2 m} \chi d \chi\right] / m !
\end{gathered}
$$

The coefficients $\alpha_{n m}$ and $\beta_{n m}$ are functions of $r^{-1}$ and are expanded up to the power $r^{-5}$. These coefficients have been evaluated and the results are as follows $(n \leq 2)$ :

$$
\begin{aligned}
\alpha_{00} & =\frac{1}{8} \pi\left(4 r^{-2}+r_{r}^{-4}+\cdots\right), \quad \alpha_{10}=\frac{1}{2} \pi r^{-2}, \\
\alpha_{20} & =\frac{1}{8} \pi\left(4 r^{-2}-r^{-4}+\cdots\right), \\
\alpha_{21} & =\frac{1}{8} \pi\left(r^{-4}+\cdots\right), \\
\beta_{00} & =(\pi / 48)\left(24 r^{-1}-16 r^{-2}-6 r^{-3}-r^{5}+\cdots\right), \\
\beta_{10} & =(\pi / 60)\left(30 r^{-1}\right. \\
& \left.-20 r^{-2}-15 r^{-3}+4 r^{-4}+\cdots\right), \\
\beta_{11} & =(\pi / 120)\left(15 r^{-3}-8 r^{-4}+\cdots\right), \\
\beta_{21} & =(\pi / 120)\left(15 r^{-3}-8 r^{-4}-5 r^{-5}+\cdots\right) .
\end{aligned}
$$

The remaining moment integral ${ }^{2} g_{3}^{n}$ turns out to be the most difficult one to cope with. To facilitate the integration we introduce the Cartesian coordinates $\left(r_{0}, t_{0}, t_{0}^{\prime}\right)$ where $r_{0}$ is the unit vector pointing from center of the sphere to the point of interest; $t_{0}$ is the unit vector, perpendicular to $r_{0}$, lying in the plane containing $r_{0}$ and $s_{0}$; $t_{0}^{\prime}$ is the unit vector perpendicular to both $r_{0}$ and $t_{0}$. In terms of the new vectors, we obtain, after a lengthy algebraic manipulation, the ${ }^{l} g_{3}^{n}$ in the following form:

$$
\begin{aligned}
{ }^{l} g_{3}^{n} & =2 \pi^{-\frac{3}{2}} \Gamma\left(\frac{1}{2}\right) \sum_{j=0}^{n} \sum_{m=0}^{N(j / 2)} \Gamma\left(m+\frac{1}{2}\right) e^{-s^{2}} \mathbf{r}_{0}^{n-i} \mathbf{t}_{0}^{i-2 m} \mathbf{t}_{0}^{\prime 2 m} \\
& \cdot \int_{0}^{\infty} e^{-c^{2}} c^{n+l+2} d c \int_{0}^{s \operatorname{in}^{-1}\left(r^{-1}\right)} d \chi \\
& \cdot \exp [-2 s c \cos \theta \cos \chi] \sin ^{i+1} \chi \cos ^{n-i} \chi \\
& \cdot\left(\frac{d^{j-2 m}}{d z^{i-2 m}} \sum_{k=0}^{\infty} \frac{(z / 2)^{2 k}}{k !(k+m) !}\right)_{z=2 \sin \sin x \sin \theta}
\end{aligned}
$$

Although the expression (4.21) appears very complicated, by expanding the coefficient in powers of $r^{-1}$, we found that the lower order of $t_{0}$ and $t_{0}^{\prime}$ always accompany with the lower orders of $r^{-1}$ as shown in (4.21). Hence for any order of $n$, there will be only a few terms of the low order $t_{0}$ and $\mathfrak{t}_{0}^{\prime}$ assuming importance in the expansion. It implies that the moment integrals ${ }^{l} g_{3}^{n}$ are predominantly r-dependent functions.

Again the moments of ${ }^{l} g_{3}^{n}$ up to $n=2$ and the expansion in power series of $r^{-1}$ to the fifth order are evaluated and presented as follows:

$$
\begin{aligned}
{ }^{0} g_{3}^{0}= & \frac{1}{4}(\pi)^{-\frac{1}{2}} e^{-8^{3}}\left[4 r^{-2} f_{2}\right. \\
& \left.+r^{-4}\left(f_{2}+2 s \cos \theta f_{3}+2 s^{2} \sin ^{2} \theta f_{4}\right)\right], \\
{ }^{2} g_{3}^{0}= & \frac{1}{4}(\pi)^{-\frac{1}{2}} e^{-8^{2}}\left[4 r^{-2} f_{4}\right. \\
& \left.+r^{-4}\left(f_{4}+2 s \cos \theta f_{5}+2 s^{2} \sin ^{2} \theta f_{6}\right)\right], \\
{ }^{0} g_{3}^{1}= & \frac{1}{4}(\pi)^{-\frac{1}{2}} e^{-s^{2}}\left[4 r^{-2} f_{3}\right. \\
& \left.+r^{-4}\left(2 s \cos \theta f_{4}+2 s^{2} \sin ^{2} \theta f_{5}\right)\right] \mathbf{r}_{0} \\
& +\frac{1}{2}(\pi)^{-\frac{1}{2}} e^{-8^{2}} s \sin \theta \mathbf{t}_{0} r^{-4} f_{4}, \\
{ }^{0} g_{3}^{2}= & \frac{1}{4}(\pi)^{-\frac{1}{2}} e^{-s^{2}}\left[4 r^{-2} f_{4}\right. \\
& \left.+r^{-4}\left(-f_{4}+2 s \cos \theta f_{5}+2 s^{2} \sin ^{2} \theta f_{6}\right)\right] r_{0}^{2} \\
& +\frac{1}{4}(\pi)^{-\frac{1}{2}} e^{-8^{2}} s \sin \theta r^{-4} f_{5} \mathbf{t}_{0} \mathbf{r}_{0} / 2 \\
& +(\pi)^{-\frac{1}{2}} e^{-8^{2}} r^{-4} f_{4}\left(\mathbf{t}_{0}^{2}+\mathbf{t}_{0}^{2}\right),
\end{aligned}
$$

where

$$
\begin{aligned}
& f_{n}(s \cos \theta)=\int_{0}^{\infty} e^{-c^{2}} c^{n} e^{-2 s c \cos \theta} d c \\
& =\sum_{m} \frac{(-1)^{m} 2^{m-1}(s \cos \theta)^{m}}{m !} \Gamma\left(\frac{n+m+1}{2}\right)
\end{aligned}
$$




\section{General Expression for the Collisional Distribution $E(\mathrm{r}, \mathrm{c})$}

Finally the expression for the collisional distribution $E(\mathbf{r}, \mathbf{c})$ can be given in computable form. It is noted that the first nonzero term in the expansion for $E(\mathbf{r}, \mathrm{c})$ is the second-order term because both $\left[H^{(0)}\right]$ and $\left[H^{(1)}\right]$ vanish. For $n=2$ the possible $j$ and $m$ are as follows:

$$
(j, m)=(1,0),(2,0), \text { and }(2,1) .
$$

With these values of $j, m$ we obtain

$$
\begin{aligned}
a_{i j}^{(2)}(\mathbf{r})= & {\left[{ }^{0} g^{2}{ }^{0} f^{0}-{ }^{0} g^{1}{ }^{0} f^{1}+{ }^{0} g^{0}{ }^{0} f^{2}+{ }^{0} g^{2}{ }^{0} g^{0}\right.} \\
& \left.-\frac{1}{2}^{0} g^{1}{ }^{0} g^{1}\right]\left(2 B_{20}-B_{10}-B_{21}\right)+\left[{ }^{2} g^{0}{ }^{0} f^{0}\right. \\
& \left.-2{ }^{0} g^{1} \cdot{ }^{0} f^{1}+{ }^{0} g^{0}{ }^{2} f^{0}+{ }^{2} g^{0}{ }^{0} g^{0}-{ }^{0} g^{1} \cdot{ }^{0} g^{1}\right] \mathbf{\delta} .
\end{aligned}
$$

The evaluation of $B_{20}, B_{10}, B_{21}$ for the Maxwellian molecules gives ${ }^{13}$

$$
2 B_{20}-B_{10}-B_{21}=-1.0562 \text {. }
$$

\section{CONTRIBUTION OF MOMENTUM FLUX BY THE COLLIDED MOLECULES}

\section{A. Formulation of Sphere Drag in an Almost-Collisionless Flow}

From the use of the collisional distribution $E(\mathbf{r}, \mathrm{c})$ we can evaluate the momentum flux to the sphere contributed by the first-order collisional effect. This constitutes, of course, an additional term to the sphere drag due to the free molecules. Note that the rate of change of the molecular distribution in the neighborhood, $\mathbf{q}, \mathbf{q}+d \mathbf{q}$ (see Fig. 2) with velocities lying between $\mathbf{c}, \mathbf{c}+d \mathbf{c}$ is

$$
0.075(d / \lambda) E(\mathbf{q}, \mathbf{c}) d \mathbf{q} d \mathbf{c},
$$

where $q$ denotes the position of point $Q$; the origin of the vector $\mathrm{q}$ is at point $P$ on the spherical surface (see Fig. 2). The direction of the velocity vector $c$ at point $Q$ is specified such that it extends from $Q$ to intersect the surface of the sphere at $P$; hence

$$
\mathrm{c}=-c \mathrm{PQ} /|\mathrm{PQ}|=-c \mathrm{q}_{0} \text {. }
$$

Furthermore, the molecules with velocity c originating from $Q$ and intersecting a surface element $d \sigma$ at $P$, lie in

$$
d \mathrm{c}=c^{2} d c \cos \tau d \theta / q^{2},
$$

where $\tau$ denotes the angle between $\mathrm{q}$ and the normal $\mathrm{n}_{0}$ to the surface at $P$. From the use of Eqs. (5.1) and (5.2) we obtain the change of the incident

${ }^{13}$ J. C. Maxwell, Collected Works (Dover Publications, Inc., New York). molecular flux and momentum flux to a unit area at $P$,

$$
\begin{gathered}
N(P)=0.075 \frac{d}{\lambda} \int_{R} d \mathbf{q} \int_{0}^{\infty} d c c^{2} E\left(\mathbf{q},-c \mathbf{q}_{0}\right) \frac{\cos \tau}{q^{2}} \tau \\
M_{i}(P)=-0.075 m \frac{d}{\lambda} \int_{R} d \mathbf{q} \\
\cdot \int_{0}^{\infty} d c c^{3} E\left(\mathbf{q},-c \mathbf{q}_{0}\right) \mathbf{q}_{0} \frac{\cos \tau}{q^{2}},
\end{gathered}
$$

respectively. The domain $R$ refers to the semi-infinite region bounded by the plane tangent to the surface at $P$ (see Fig. 2). The momentum flux taken from the body by the reflected molecules of the amount $N(P)$ is, assuming diffuse reflection,

$$
M_{r}(P)=\frac{1}{2} \pi^{\frac{1}{2}} m N(P) \mathbf{n}_{0} .
$$

The net change of momentum flux for an area $d \sigma$ around the point $P$ is

$$
\left[M_{i}(P)-M_{i}(P)\right] d \sigma
$$

and the sphere drag contributed by the first-order collisional effect, in dimensionless drag coefficient

$$
\begin{aligned}
& \Delta C_{D}=0.6 \frac{d}{\lambda}\left(\pi d^{2} s\right)^{-1} \int_{\text {sphere }} d \sigma \\
& \cdot\left[M_{i}(P)-\frac{1}{2} \pi^{\frac{k}{2}} m N(P) \mathbf{n}_{0}\right]
\end{aligned}
$$

[Drag coefficient is defined as the ratio of the drag force in question and the total dynamic pressure based on the sphere cross sectional area $\frac{1}{4} \pi d^{2}$ and the free-stream density and velocity.]

\section{B. Computation}

In order to facilitate the integration processes in the formulations of the sphere drag, we must introduce a coordinate transformation from the $\left(\mathbf{r}_{0}, \mathbf{t}_{0}, \mathbf{t}_{0}^{\prime}\right)$ -system, on which the moment integrals ${ }^{l} g^{n}$ have been prescribed, to a new $(q, \tau, \sigma)$-system in terms of which the sphere drag will be conveniently expressed. Referring to Fig. $2, \mathrm{k}$ is in the same direction as $\mathbf{n}_{0} \cdot \mathbf{i}$ and $\mathbf{k}$ are coplanar with $\mathbf{s}_{\mathbf{0}}$ and $\mathbf{n}_{0}$ while $\mathbf{j}$ is normal to them. In terms of these unit vectors we express the following quantities:

$$
\begin{aligned}
\mathbf{q}= & q \sin \tau \cos \sigma \mathbf{i}+q \sin \tau \sin \sigma \mathbf{j}+q \cos \tau \mathbf{k}, \\
\mathbf{r}= & q \sin \tau \cos \sigma \mathbf{i} \\
& +q \sin \tau \sin \sigma \mathbf{j}+(1+q \cos \tau) \mathbf{k}, \\
\mathbf{s}_{0}= & -\sin \Phi \mathbf{i}-\cos \Phi \mathbf{k}, \\
\mathbf{c}_{0}= & -\mathbf{q}_{0} .
\end{aligned}
$$


Integrals (5.3) and (5.4) become, respectively,

$$
\begin{aligned}
N(\Phi)= & \int_{0}^{\infty} d q \int_{0}^{\pi / 2} d \tau \sin \tau \cos \tau \\
& \cdot \int_{0}^{2 \pi} d \sigma \int_{0}^{\infty} d c c^{2} a_{i j}^{(2)} H_{i j}^{(2)}, \\
M(\Phi)= & \int_{0}^{\infty} d q \int_{0}^{\pi / 2} d \tau \sin \tau \cos \tau \\
& \cdot \int_{0}^{2 \pi} d \sigma \int_{0}^{\infty} d c c^{2} m\left(\mathbf{s}_{0} \cdot \mathbf{c}\right) a_{i j}^{(2)} H_{i j}^{(2)} .
\end{aligned}
$$

After the substitution of the contracted result of $H^{(2)} a^{(2)}$ in (5.9) and (5.10), and the use of (5.8), the general term of which appear of the following type:

$$
q^{k} \cos ^{l} \tau \sin ^{m} \tau \cos ^{\alpha} \Phi \sin ^{\beta} \Phi \cos ^{\gamma} \sigma / r^{p} .
$$

Let

$$
\begin{aligned}
I_{p \alpha \beta \gamma}^{k l m}= & \int_{0}^{\infty} d q \int_{0}^{\pi / 2} d \tau \int_{0}^{\pi} d \Phi \int_{0}^{2 \pi} d \sigma r^{-p} \\
& \cdot q^{k} \cos ^{l} \tau \sin ^{m} \tau \cos ^{\alpha} \Phi \sin ^{\beta} \Phi \cos ^{\gamma} \sigma
\end{aligned}
$$

and note that $I_{p \alpha \beta}^{k l m}$ vanishes after integration with respect to $\sigma$ with odd $\gamma$; with even $\gamma$ and next integration for $\Phi, I_{p \alpha \beta \gamma}^{k l m}$ again vanishes with odd $\alpha$.

In addition to the functions of (5.12), we need some more building blocks to facilitate the computation of sphere drag which are defined as follows.

Let $\omega=\mathbf{s}_{0} \cdot \mathbf{r}_{0} ; y=\mathbf{s}_{0} \cdot \mathbf{c}_{0} ; z=\mathbf{c}_{0} \cdot \mathbf{r}_{0}$, we define

$$
\begin{aligned}
A_{i j}= & \int_{0}^{\infty} d q \int_{0}^{\pi / 2} d \tau \int_{0}^{\pi} d \Phi \int_{0}^{2 \pi} d \sigma \\
& \cdot y \omega^{i} r^{-i} \sin \Phi \sin \tau \cos \tau \quad(i, \text { odd }), \\
A_{i j}^{*}= & \int_{0}^{\infty} d q \int_{0}^{\pi / 2} d \tau \int_{0}^{\pi} d \Phi \int_{0}^{2 \pi} d \sigma \\
& \cdot \omega^{i} r^{-j} \sin \Phi \cos \Phi \sin \tau \cos \tau \quad(i, \text { odd }) .
\end{aligned}
$$

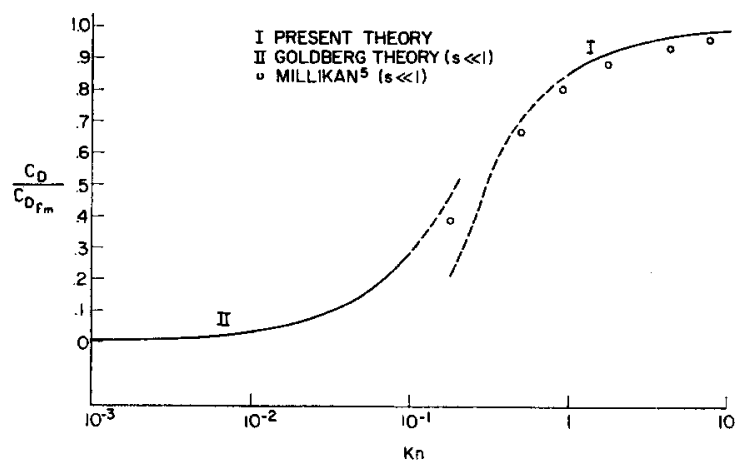

FIG. 3. Drag coefficient of a sphere.

Similarly we may define $B_{i i}, D_{i i}$, and $G_{i j}$ by replacing $y$ in (5.13) with $z y^{2}$ (with $i$, even), $z^{2} y$ (with $i$, odd) and $y^{3}$ (with $i$, odd) respectively; also define $B_{i j}^{*}, D_{i j}^{*}$, and $G_{i i}^{*}$ by replacing $\omega^{i}$ in (5.14) with $z y \omega^{i}$ (with $i$, even), $z^{2} \omega^{i}$ (with $i$, odd), and $y^{2} \omega^{i}$ (with $i$, odd), respectively.

\section{Results}

The double integrals

$$
I_{p}^{k l m}=\int_{0}^{\infty} d q \int_{0}^{\pi / 2} d \tau q^{k} \cos ^{l} \tau \frac{\sin ^{m}}{r^{\bar{\nu}}} \tau
$$

obtained from (5.12), after elementary integrations, are numerically integrated on the IBM $7090 \mathrm{com}$ -

\begin{tabular}{|c|c|c|c|c|c|}
\hline $\begin{array}{l}A_{12}= \\
A_{32}= \\
B_{02} \\
B_{24}= \\
D_{12}= \\
D_{32}= \\
G_{12}= \\
G_{32}=\end{array}$ & $\begin{array}{l}=-2.09439 \\
=-1.25658 \\
=-2.09439 \\
=-0.33504 \\
=-1.74524 \\
=-1.04704 \\
=-1.25662 \\
=-0.83773\end{array}$ & $\begin{array}{l}A_{13}=-1.04719 \\
A_{34}=-0.41880 \\
B_{04}=-0.69813 \\
B_{42}=-0.77781 \\
D_{13}=-0.78530 \\
D_{34}=-0.29309 \\
G_{13}=-0.62832 \\
G_{34}=-0.26324\end{array}$ & $\begin{array}{l}A_{14}=-0.69813 \\
A_{35}=-0.31408 \\
B_{05}=-0.52358 \\
B_{44}=-0.22733 \\
D_{14}=-0.48859 \\
D_{35}=-0.20931 \\
G_{14}=-0.41885\end{array}$ & $\begin{array}{l}A_{15} \\
B_{22} \\
B_{45} \\
D_{15} \\
G_{15}\end{array}$ & $\begin{array}{l}=-0.52358 \\
=-1.11696 \\
=-0.16451 \\
=-0.34896 \\
=-0.31415\end{array}$ \\
\hline $\begin{array}{l}A_{12}{ }^{*}= \\
A_{32}{ }^{*}= \\
B_{02}{ }^{*}= \\
B_{22^{*}}{ }^{*}= \\
D_{12}{ }^{*}= \\
D_{32}{ }^{*}= \\
G_{12}{ }^{*}= \\
G_{32}{ }^{*}=\end{array}$ & $\begin{array}{l}=-2.09436 \\
=-1.25655 \\
=-1.39626 \\
=-0.33347 \\
=-1.62889 \\
=-0.97723 \\
=-0.97738 \\
=-0.69811\end{array}$ & $\begin{array}{l}A_{13}{ }^{*}=-1.19542 \\
A_{34}{ }^{*}=-0.51400 \\
B_{04}{ }^{*}=-0.46541 \\
B_{42^{*}}=-0.67809 \\
D_{13}{ }^{*}=-0.82237 \\
D_{34}{ }^{*}=-0.33440 \\
G_{13}{ }^{*}=-0.51834 \\
G_{34}{ }^{*}=-0.24640\end{array}$ & $\begin{array}{l}A_{14^{*}}=-0.85682 \\
A_{35}{ }^{*}=-0.40436 \\
B_{05^{*}}=-0.34905 \\
B_{44}{ }^{*}=-0.22621 \\
D_{14}{ }^{*}=-0.54361 \\
D_{35}{ }^{*}=-0.24179 \\
G_{14}{ }^{*}=-0.35753\end{array}$ & $\begin{array}{l}A_{15}{ }^{*} \\
B_{22}^{*} \\
B_{45}^{*} \\
D_{15}^{*} \\
G_{15}^{*}\end{array}$ & $\begin{array}{l}=-0.67410 \\
=-0.93080 \\
=-0.16737 \\
=-0.40314 \\
=-0.27446\end{array}$ \\
\hline
\end{tabular}
puter for integer indices in the ranges: $0<k \leq 5,1 \leq$ $l \leq 8,1 \leq m \leq 7,3 \leq p \leq 10$ with $k+1<p$.

The basic integrals $A_{i j}, A_{i j}^{*}, B_{k_{i}}, B_{k i}^{*}, D_{i j}, D_{i i}^{*}$, $G_{i j}$, and $G_{i j}^{*}$ with integer indices in ranges $i=1,3$, $j=2$ to 5 and $k=0,2,4$ are computed. These basic integrals are the building blocks which enable us to calculate the sphere drag. The computed functions are tabulated in Table I.

The drag coefficient of a sphere in flows of an almost free molecule, normalized by the correspond-

TABLE I. Values of the basic integrals. 
Table II. Coefficients $B(s)$.

\begin{tabular}{cccccccccccccccc}
\hline \hline \multirow{2}{*}{$10^{-5}$} & $10^{-2}$ & 0.1 & 0.2 & 0.3 & 0.4 & 0.5 & 0.6 & 0.7 & 0.8 & 0.9 & 1 & 0.154 \\
\hline$B(s)$ & 0.149 & 0.149 & 0.150 & 0.150 & 0.151 & 0.153 & 0.154 & 0.155 & 0.156 & 0.154 & 0.152 & 0.148 & \\
\hline
\end{tabular}

ing value for the free molecular flows can be expressed as follows ${ }^{14}$ :

$$
C_{\mathrm{D}} / C_{\mathrm{D}_{\mathrm{f}} \mathrm{s}}=1-B(s) / \mathrm{Kn}
$$

where $B(s)$ is given in Table II.

The results of computations of the aerodynamic drag for a sphere in an almost collisionless flow are shown in Fig. 3. The drag components contributed by the incident species $\left(M_{\mathrm{i}}\right)$ and the reflected species $(N)$ respectively are also shown in Fig. 4 . All the calculations are based upon the assumed condition that the sphere temperature is equal to the freestream temperature, and perfect diffuse reflection prevails. Although it is difficult to prove the convergence of the expansion, we are able to establish the rapid decrease of the absolute values of the succeeding terms in all the expansions when the speed ratio $s$ is not much larger than 1 .

It is significant to note that the theoretical result calculated for a very small value of $s\left(s=10^{-5}\right)$ agrees with Millikan's measured values ${ }^{15}$ at corresponding $s$ over a wide range of Knudsen numbers $(0.5<\lambda / d<$ $10)$. It is also noted that over a wide range of speed ratios $(s<1.0)$ the drag coefficient ratio $C_{\mathrm{D}} / C_{\mathrm{D}_{\mathrm{fm}}}$ $\left(C_{D_{f m}}\right.$ denotes the drag coefficient of the sphere in which the free molecules flow at the same speed ratio) depends only on the Knudsen number $(\lambda / d)$ in this first-order approximation (see Table II). Even for $1<s<1.5$ the computed results for $C_{\mathrm{D}} / C_{\mathrm{D}_{\mathrm{fm}}}$ show only slight dependence on $s$. We feel however that to vindicate this conclusion with respect to the range $s>1$, higher-order terms of $s$ in the expansions must be included.

\section{DISCUSSIONS AND CONCLUSIONS}

The classification of rarefied flows, e.g., the continuum, transition, free molecular, has been traditionally based on values of the Knudsen number, a ratio of the mean free path, $\lambda$, and a characteristic dimension of the body, $d$. In view of the asymptotic solutions of the Boltzmann equation we can give a different viewpoint to the flow regimes.

Consider the flow field around a body. Within a distance much less than a mean free path from the

\footnotetext{
${ }^{14}$ The suggestion of the reviewer is appreciated.

${ }_{15}$ In this comparison the same expression for the mean free path is used in calculating Knudsen numbers.
}

Fig. 4. Drag contributions by the reflected and the incident molecules.

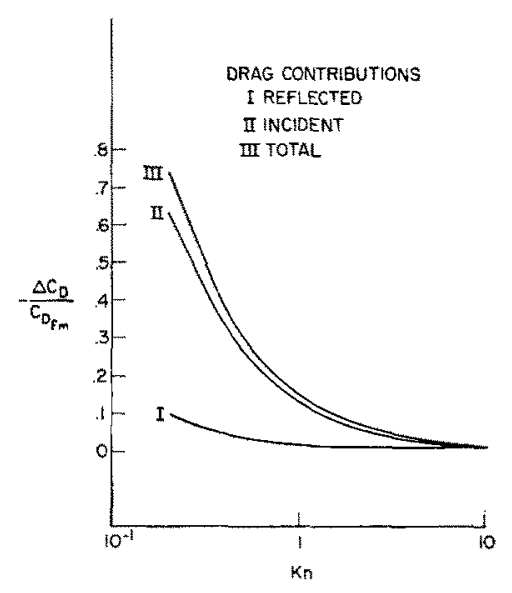

body the molecular distribution would exhibit the features of quasi-free molecular flow since the collisions between the streams incident on and reflected from the surface is dominated by the collisions between the incident stream and the surface. On the other hand, at distances of many free paths away from the body the moleculer distribution is almost locally Maxwellian, provided that sufficiently large volumes are used for sampling; hence, it can be treated as quasi-continuum. At the intermediate distance from the body, the molecular distribution will deviate from both the asymptotic solutions mentioned above. The significance of each of the three flow regimes pertaining to a given flow must be determined by the Knudsen number in question.

Although there is little doubt that the transition flow structure in a monatomic gas is contained in the Boltamann equation, we cannot treat the transition flow as an entity because the contemporary solutions to this flow regime are valid only either near continuum or near-free molecular flow. In the former class there is Goldberg's solution to the 13-moment equations which are linearized for the problem of a slowly moving sphere. Contrary to Grad's remark, ${ }^{16}$ Goldberg's sphere drag solution cannot be expected to cover the whole range of mean free paths because it would not be a meaningful approximation to the free or near-free molecular flows (see Fig. 3).

In the latter class there is a valuable solution by

${ }^{16}$ H. Grad, in Handbuch der Physik, edited by S. Flügge (Springer-Verlag, Berlin, 1958), Vol. 12, p. 292. 
Szymanski ${ }^{4}$ which is a first-order Knudsen iteration of the Boltzmann equation. Like Goldberg, Szymanski also limits his discussion to the slowly moving sphere such that linearization of the disturbance effect can be used. Unfortunately, we found two gross errors in Szymanski's development and hence have serious reservation about the validity of his results. [One of these is in his equation (7.10), another is Eq. (7.14) in Ref. 4.] In any event, his theory is developed for the flows of extremely low speeds only.

At the other extreme of speed ratios $(s \gg 1)$ there are theories of sphere drag based on either Boltzmann equation with simplified collision integral ${ }^{2}$ or collision statistics. ${ }^{3}$ In both cases approximate kinetic models for collisions have been introduced. On the experimental side there is hardly any data available for such high Knudsen numbers. Besides, much of the sphere drag measured at intermediate Knudsen numbers is made with unknown surface temperature on which the sphere drag strongly depends.

The present theory based on the exact ${ }^{17}$ Boltzmann Collision integral for Maxwellian molecules is formulated for high Knudsen number $(\mathrm{Kn} \geq 1)$ and a speed range $0<s<1$; the upper limit for $s$ is not of the cutoff nature. In fact, it can be extended to higher values of $s$ with more terms in the expansions. The effect on the sphere drag due to the use of an artificial molecular model, such as the Maxwellian molecules is difficult to estimate.

The lack of sphere drag measurements at corresponding Knudsen number and surface temperature makes it impossible to ascertain the accuracy of the present theory; nevertheless its close agreement (within 5\%) with Millikan's experimental results at the low speeds in a wide range of Knudsen numbers does appear encouraging. A composite plot of sphere drag against Knudsen numbers including the continuum range is given in Fig. 3 to lend some support to the present theory.

${ }_{17}$ The only approximation introduced is at representation of the molecular flux of the reflected molecules other than the truncation of the Hermite expansion.
Much of the contemporary studies of flows, at moderately high Knudsen numbers, with exact Boltzmann equation approach are limited to simple internal flows such as Couette flows, etc. Should the present results be considered favorable, it could suggest that the method of the present approach might have opened a new effective avenue to the mysterious regime of transition flows.

\section{ACKNOWLEDGMENT}

This work was supported in part by the Upper Atmospheric Physics Laboratory Geophysics Research Directorate, AFCRL, the Phoenix Memorial Research Grant of The University of Michigan, and NASA Research Grant NsG660.

\section{APPENDIX I. DETERMINATION OF $\alpha(\mathrm{s})$ AND $\beta(\mathrm{s})$}

The equivalent number density of the reflected molecules at point $P$ on the surface [see Eq. (3.2)]

$n(P)=\exp \left[-(s x)^{2}\right]-\pi^{\frac{1}{2}} s x+\pi^{\frac{1}{2}} s x \operatorname{erf}(s x) \equiv n_{1}$,

where $x=\mathbf{n}_{0} \cdot \mathbf{s}_{0}$ is approximated by the linear function

$$
n(P)=\alpha s x+\beta \equiv n_{2},
$$

such that the total number of molecules reflected from the semispherical surface on the upstream side based on (A1) is equal to that based on (A2); similarly for the reflected molecules for the downstream semispherical surface, i.e.,

$\int_{0}^{-1} n_{1} d x=\int_{0}^{-1} n_{2} d x, \quad \int_{0}^{+1} n_{1} d x=\int_{0}^{+1} n_{2} d x$.

Performing the integrations and solving for $\alpha$ and $\beta$ we obtain

$\alpha=-\pi^{\frac{1}{2}}$

$\beta=\pi^{\frac{1}{2}}\left[\left(1+2 s^{2}\right)\right.$ erf $\left.s+2 s \pi^{-\frac{1}{2}} \exp \left(-s^{2}\right)\right](4 s)^{-1}$. 